\title{
Food Flavor Perception as Expressed via Sensory Spectrograph
}

\author{
Naomi Sano, Ayaka Miyamoto, Mao Igasaki, Shiori Itoh, Haruna Ohkaji, Yoshie Yamagata, \\ Jun Kayashita, Sumi Sugiyama, Yoshiaki Sugawara* \\ Department of Health Science, Prefectural University of Hiroshima, Hiroshima, Japan \\ Email: *sugawara@pu-hiroshima.ac.jp
}

Received 28 December 2015; accepted 14 February 2016; published 17 February 2016

Copyright @ 2016 by authors and Scientific Research Publishing Inc.

This work is licensed under the Creative Commons Attribution International License (CC BY). http://creativecommons.org/licenses/by/4.0/

(c) (i) Open Access

\begin{abstract}
In our previous studies, we examined the relationship between changes in mood, verbal (semantic) behavior, and non-verbal (skin temperature) activity induced by inhalation of essential oil fragrances, as well as linalool and its enantiomers. Sensory evaluation was a key component of these studies. We have found that perceived sensory attributes reported by participants can be represented via sensory spectrograph: A bar graph where the mean of the impression is plotted against descriptors of the setting for the semantic impression. In this paper, we present our latest attempts at assessing the taste of food using a sensory spectrograph. We conducted two studies: One in which participants assessed the taste of cookies with or without bean curd lees and one in which participants evaluated the taste of Miso soup and Sumashi soup as a function of salty concentration and soup stock consistency.
\end{abstract}

\section{Keywords}

Cookies with or without Bean Curd Lees, Food Flavor, Miso Soup, Sensory Evaluation, Sumashi Soup

\section{Introduction}

Sensory evaluation can be used to measure elements of consciousness. As a result, it has been a key element of experimental and mathematical psychology (Coombs, 1964; Guilford, 1954; Kling \& Riggs, 1972; Stevens, 1951; Torgerson, 1958). Sensory experiences can be reported using verbal (semantic) methods.

Previously, we conducted a series of studies to investigate the psychophysiological effects of inhaling essential oils, as well as linalool and its enantiomers $((R)-(-)-,(S)-(+)-$, and $(R, S)-( \pm)$-forms). Specifically, we ex-

*Corresponding author.

How to cite this paper: Sano, N., Miyamoto, A., Igasaki, M., Itoh, S., Ohkaji, H., Yamagata, Y., Kayashita, J., Sugiyama, S., \& Sugawara, Y. (2016). Food Flavor Perception as Expressed via Sensory Spectrograph. Psychology, 7, 223-237. 
amined changes in mood, as well as verbal (semantic) and non-verbal (skin temperature) activity in humans after exposure to fragrant compounds (Satoh \& Sugawara, 2003; Sugawara, 2001, 2008; Sugawara \& Kawasaki, 2000; Sugawara et al., 1998a, 1998b, 1999, 2000, 2003, 2006, 2008, 2009a, 2009b, 2013, 2015a, 2015b; Yamagata \& Sugawara, 2014). Meanwhile, sensory evaluation has been a key component of our research activities (Sugawara, 2008; Sugawara et al., 2009a, 2009b, 2013, 2015a, 2015b; Yamagata \& Sugawara, 2014).

As a measure (sensory profile) of perceived odor quality, we asked participants to complete a sensory questionnaire after inhalation of a given aroma (Sugawara, 2008; Sugawara et al., 2009a, 2009b, 2013, 2015a, 2015b; Yamagata \& Sugawara, 2014). The questionnaire evaluated aroma perception using 13 descriptors in the form of paired contrasting adjectives: fresh - stale, soothing - activating, airy - heavy, plain - rich, natural - unnatural, elegant - unrefined, soft - strong, pleasant - unpleasant, warm - cool, comfortable - uncomfortable, woodsy - not woodsy, floral - peppery, lively - dull. The 13 descriptors were scored on an 11-point scale $(-5$ to +5$)$, with 0 as the middle score. To test the effects of the aromas on behavior, we employed the Uchida-Kraepelin test as a mental arithmetic task and a task involving listening to environmental (natural) sounds as an auditory task. The sensory evaluation test (including exposure to the aroma and completion of the questionnaire mentioned above) was conducted twice, once before and once after the task. The perceived sensory attributes of a given aroma could be represented as a function of behavioral task performance using a sensory spectrograph, which is a bar graph in which the mean of the difference in score between pre- and post-task inquiry (post minus pre) was plotted against the impression descriptors. We were able to establish the sensory attributes of the following 21 essential oils, as well as linalool and its enantiomers, as a function of the two behavioral tasks (mental arithmetic and auditory): basil, bergamot, cardamom, chamomile, cinnamon, clove, cypress, geranium, ho leaf/wood, juniper, lavender, lemon, lime, marjoram, orange, palmarosa, peppermint, rosemary, sandalwood, spearmint, and ylangylang (Sugawara et al., 2015a).

The resulting sensory spectra warranted particular interest (Sugawara et al., 2015a). For instance, we observed three types of sensory spectra. One is an upward (positive) spectrum, where the descriptors regarded as significant via a $t$-test had a positive value and were shown above the horizontal axis, signifying a positive (or favorable) correlation between the fragrance of a given aroma and the type of task with respect to the positive descriptors (i.e., "fresh", “airy”, "elegant”, "pleasant”, “comfortable”, etc.). The next is a downward (negative) spectrograph, in which negative values appeared below the axis, suggesting an unfavorable (negative) correlation between the fragrance and the type of task in terms of the negative descriptors (i.e., "stale", "heavy", "unrefined", "unpleasant", "uncomfortable", etc.). The third was a "miscellaneous" type, where half of the significant descriptors were positive and the other half were negative. The sensory spectra classified as part of the first category ("favorable" type) were seen for bergamot, peppermint, sandalwood, ylangylang, and others if they were paired with the auditory task, while the spectra belonging to the second ("unfavorable" type) were observed for bergamot, peppermint, sandalwood, ylangylang, and others if they were paired with mental arithmetic. Moreover, the spectrographs classified as part of the third ("miscellaneous" type) were found for the following pairings: cardamom/mental arithmetic, cinnamon/mental arithmetic, lemon/auditory task, orange/mental arithmetic, orange/ auditory task, and so on.

Given the opposite shape of the sensory spectrographs produced upon inhalation of bergamot, peppermint, sandalwood, or ylangylang depending on whether the participants completed the mental arithmetic or the auditory talk, our research indicates that different behavioral tasks can evoke different perceived odor quality of a given aroma.

The task-dependent responses seen in bergamot, peppermint, sandalwood, and ylangylang essential oils were also found in linalool and (RS)-( \pm -)-linalool, which is refined from commercial linalool via repeated flash column chromatography with silica gel (Sugawara et al., 2015a). Linalool and the associated refined (purified) material also produced opposite sensory spectra in a task dependent way. Additionally, a detailed inspection of the spectra of optically active linalools $((R)-(-)-,(S)-(+)-$, and $(R, S)-( \pm)$-forms) revealed that the $(R)-(-)-$ and $(S)-(+)-$ forms of linalool produce different sensory spectra when participants are engaged in mental arithmetic, but identical spectra when the auditory task is employed. Given these findings and the suggestion by Ohloff and Klein (1962) that (+)- and (-)-linalool are petitgrain-like and lavender-like, respectively, we speculated that different enantiomers of linalool may evoke distinct odor percepts in a task-dependent manner.

When assessing perceived odor quality as a function of different behavioral tasks assigned to participants, we have been able to discern nuanced individual differences in the spectrograph for each participant. This implies that our method of sensory profiling via sensory spectrograph may be practical for multimodal methodologies 
(i.e., for multimodal sensory targets).

Based on our experiences conducting sensory profiling of aromas, in this paper, we sought to assess the perception of food flavor using sensory spectrographs. We conducted two flavor studies: 1) an evaluation of the taste of cookies with or without bean curd lees; and 2) an evaluation of the taste of Miso soup and Sumashi soup as a function of salty concentration and soup stock consistency.

\section{Method}

\subsection{Recipes for Cookies with or without Bean Curd Lees}

We generated two recipes for making cookies: one with and one without bean curd lees. We found that both generally succeeded in producing "moist cookies".

First, we will describe the process for making cookies fortified with bean curd lees.

First, we preheated an oven to $180^{\circ} \mathrm{C}$. We prepared $70 \mathrm{~g}$ bean curd lees by heating in a frying pan until most of the moisture had been evaporated. We then let the bean curd lees cool for 5 minutes. In a mixing bowl, we used electric beaters to cream $70 \mathrm{~g}$ of salt-free butter at room temperature. We then added $50 \mathrm{~g}$ sugar and blended well until the mixture turned white. We then separated 2 eggs, discarding the whites. We added the egg yolks to the mixing bowl one at a time, mixing well between eggs. We then added the bean curd lees along with $100 \mathrm{~g}$ sifted flour, and mixed the batter well using a spatula. Following that, we added $50 \mathrm{~g}$ raisins and then $6 \mathrm{~g}$ ground tea leaf, mixing well between the two. We then used a spoon to round out sections of dough, which were placed on to a parchment-lined baking sheet and baked for 22 minutes.

We used the same recipe for cookies without bean curd lees, except that the bean curd lees were replaced by an equivalent amount of flour. The recipe made approximately 26 cookies.

\subsection{Brewing Instructions for 12 Varieties of Miso Soup and Sumashi Soup}

Table 1 summarizes our brewing instructions for twelve varieties of Miso soup (Table 1(a)) and Sumashi soup

Table 1. Summary of brewing instructions for 12 varieties of Miso soup and Sumashi soup. The varieties of both Miso soup and Sumashi soup include three concentrations of salt (i.e., $0.5 \%, 0.7 \%$, and $0.9 \%$ ), and each salt variant has four types of soup stock consistency (i.e., $0 \%, 0.1 \%, 0.2 \%$, and $0.3 \%$ ).

(a)

\begin{tabular}{|c|c|c|c|c|c|c|}
\hline & & \multirow{3}{*}{ ingredients } & \multicolumn{4}{|c|}{ soup stock consistancy } \\
\hline & & & 0\% & $0.1 \%$ & $0.2 \%$ & $0.3 \%$ \\
\hline & & & $\begin{array}{l}\text { (without soup } \\
\text { stock) }\end{array}$ & $\begin{array}{l}\text { (half amount of } \\
\text { ordinary used) }\end{array}$ & $\begin{array}{l}\text { (amount ordinary } \\
\text { used) }\end{array}$ & (a little more serving) \\
\hline \multirow{12}{*}{ 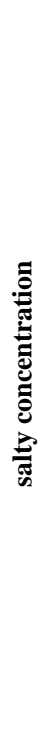 } & \multirow{4}{*}{$0.5 \%$} & $\begin{array}{l}\text { soup stock cube } \\
\text { (granulated) }\end{array}$ & $0 \mathrm{~g}$ & $2.1 \mathrm{~g}$ & $4.2 \mathrm{~g}$ & $6.3 \mathrm{~g}$ \\
\hline & & soybeam paste & $17.82 \mathrm{~g}$ & $17.82 \mathrm{~g}$ & $17.82 \mathrm{~g}$ & $17.82 \mathrm{~g}$ \\
\hline & & $\begin{array}{l}\text { the content of salt originated from } \\
\text { soybean paste used (estimated) }\end{array}$ & $1.853 \mathrm{~g}$ & $1.853 \mathrm{~g}$ & $1.853 \mathrm{~g}$ & $1.853 \mathrm{~g}$ \\
\hline & & cooking salt & $2.646 \mathrm{~g}$ & $1.764 \mathrm{~g}$ & $0.882 \mathrm{~g}$ & $0 \mathrm{~g}$ \\
\hline & \multirow{4}{*}{$0.7 \%$} & soup stock cube (granulated) & $0 \mathrm{~g}$ & $2.1 \mathrm{~g}$ & $4.2 \mathrm{~g}$ & $6.3 \mathrm{~g}$ \\
\hline & & soybeam paste & $17.82 \mathrm{~g}$ & $17.82 \mathrm{~g}$ & $17.82 \mathrm{~g}$ & $17.82 \mathrm{~g}$ \\
\hline & & $\begin{array}{l}\text { the content of salt originated from } \\
\text { soybean paste used (estimated) }\end{array}$ & $1.853 \mathrm{~g}$ & $1.853 \mathrm{~g}$ & $1.853 \mathrm{~g}$ & $1.853 \mathrm{~g}$ \\
\hline & & cooking salt & $4.446 \mathrm{~g}$ & $3.564 \mathrm{~g}$ & $2.682 \mathrm{~g}$ & $1.800 \mathrm{~g}$ \\
\hline & \multirow{4}{*}{$0.9 \%$} & $\begin{array}{l}\text { soup stock cube } \\
\text { (granulated) }\end{array}$ & $0 \mathrm{~g}$ & $2.1 \mathrm{~g}$ & $4.2 \mathrm{~g}$ & $6.3 \mathrm{~g}$ \\
\hline & & soybeam paste & $17.82 \mathrm{~g}$ & $17.82 \mathrm{~g}$ & $17.82 \mathrm{~g}$ & $17.82 \mathrm{~g}$ \\
\hline & & $\begin{array}{l}\text { the content of salt originated from } \\
\text { soybean paste used (estimated) }\end{array}$ & $1.853 \mathrm{~g}$ & $1.853 \mathrm{~g}$ & $1.853 \mathrm{~g}$ & $1.853 \mathrm{~g}$ \\
\hline & & cooking salt & $7.092 \mathrm{~g}$ & $5.364 \mathrm{~g}$ & $4.482 \mathrm{~g}$ & $3.600 \mathrm{~g}$ \\
\hline
\end{tabular}


(b)

\begin{tabular}{|c|c|c|c|c|c|c|}
\hline & & \multirow{3}{*}{ ingredients } & \multicolumn{4}{|c|}{ soup stock consistancy } \\
\hline & & & $\mathbf{0} \%$ & $0.1 \%$ & $0.2 \%$ & $0.3 \%$ \\
\hline & & & (without soup stock) & $\begin{array}{l}\text { (half amount of } \\
\text { ordinary used) }\end{array}$ & $\begin{array}{l}\text { (amount ordinary } \\
\text { used) }\end{array}$ & (a little more serving) \\
\hline \multirow{12}{*}{ 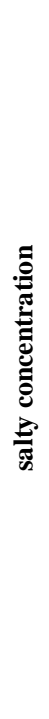 } & \multirow{4}{*}{$0.5 \%$} & soup stock cube (granulated) & $0 \mathrm{~g}$ & $2.1 \mathrm{~g}$ & $4.2 \mathrm{~g}$ & $6.3 \mathrm{~g}$ \\
\hline & & soy sauce & $3.096 \mathrm{~g}$ & $3.096 \mathrm{~g}$ & $3.096 \mathrm{~g}$ & $3.096 \mathrm{~g}$ \\
\hline & & $\begin{array}{l}\text { the content of salt originated from } \\
\text { soy sauce used (estimated) }\end{array}$ & $1.344 \mathrm{~g}$ & $1.344 \mathrm{~g}$ & $1.344 \mathrm{~g}$ & $1.344 \mathrm{~g}$ \\
\hline & & cooking salt & $2.646 \mathrm{~g}$ & $1.764 \mathrm{~g}$ & $0.882 \mathrm{~g}$ & $0 \mathrm{~g}$ \\
\hline & \multirow{4}{*}{$0.7 \%$} & soup stock cube (granulated) & $0 \mathrm{~g}$ & $2.1 \mathrm{~g}$ & $4.2 \mathrm{~g}$ & $6.3 \mathrm{~g}$ \\
\hline & & soy sauce & $6.06 \mathrm{~g}$ & $6.06 \mathrm{~g}$ & $6.06 \mathrm{~g}$ & $6.06 \mathrm{~g}$ \\
\hline & & $\begin{array}{l}\text { the content of salt originated from } \\
\text { soy sauce used (estimated) }\end{array}$ & $2.64 \mathrm{~g}$ & $2.64 \mathrm{~g}$ & $2.64 \mathrm{~g}$ & $2.64 \mathrm{~g}$ \\
\hline & & cooking salt & $2.646 \mathrm{~g}$ & $1.764 \mathrm{~g}$ & $0.882 \mathrm{~g}$ & $0 \mathrm{~g}$ \\
\hline & \multirow{4}{*}{$0.9 \%$} & soup stock cube (granulated) & $0 \mathrm{~g}$ & $2.1 \mathrm{~g}$ & $4.2 \mathrm{~g}$ & $6.3 \mathrm{~g}$ \\
\hline & & soy sauce & $9 \mathrm{~g}$ & $9 \mathrm{~g}$ & $9 \mathrm{~g}$ & $9 \mathrm{~g}$ \\
\hline & & $\begin{array}{l}\text { the content of salt originated from } \\
\text { soy sauce used (estimated) }\end{array}$ & $3.96 \mathrm{~g}$ & $3.96 \mathrm{~g}$ & $3.96 \mathrm{~g}$ & $3.96 \mathrm{~g}$ \\
\hline & & cooking salt & $2.646 \mathrm{~g}$ & $1.764 \mathrm{~g}$ & $0.882 \mathrm{~g}$ & $0 \mathrm{~g}$ \\
\hline
\end{tabular}

(Table 1(b)), including three different salt concentrations (i.e., $0.5 \%, 0.7 \%$, and $0.9 \%$ ), and four types of soup stock consistency (i.e., $0 \%, 0.1 \%, 0.2 \%$, and $0.3 \%$ ). In the table, each row (line) corresponds to each experimental run.

In preparing the twelve varieties of Miso soup (Table 1(a)), we first prepared the four samples of Miso soup outlined in the top row of Table 1(a). These variants had a fixed $0.5 \%$ salt concentration but varying soup stock consistency, ranging from $0 \%$ to $0.3 \%$ (i.e., $0 \%, 0.1 \%, 0.2 \%$ and $0.3 \%$ ). Next, we made the four samples that appear in the second row of the table. These have a constant $0.7 \%$ salt concentration but varying soup stock consistency, which ranges from $0 \%$ to $0.3 \%$. Third, we assembled the four samples of Miso soup from the bottom row of Table 1 (a), which have a constant $0.9 \%$ salt concentration but varying soup stock consistency, which ranges from $0 \%$ to $0.3 \%$. We followed this order because it coincided with the order of the experimental runs. Brewing instructions for the twelve varieties of Sumashi soup (Table 1(b)) were identical to those for Miso soup (Table 1(a)), except that we used light soy sauce to prepare Sumashi soup instead of soybean paste, which was used for Miso soup.

To make Miso soup, we first placed the required amount (grams) of granulated bonito-fish soup stock, soybean paste, and cooking salt in a $1 \mathrm{~L}$ scaled stainless steel beaker equipped with a handle. We then poured hot water (just under the boiling point) into the beaker, mixed well with a whisk, and added more water to reach $1 \mathrm{~L}$ in total. We then brewed the soup for 3 - 5 minutes. We matched the amount of cooking salt in each sample to the estimated amount of saline ingredients in the soybean paste used for each specimen. We used HONDASHI as the granulated bonito-fish soup stock and SETO-NO-HONJIO (common mineral salt originating from the water of the Seto Sea) as the cooking salt, which are both produced by AJINOMOTO Co. (Tokyo, Japan). Our soybean paste (non-additive blend of Miso fermented with barley koji and Miso fermented with rice koji) was produced by SHINJYO MISO Co. (Hiroshima, Japan), and the light soy sauce was from HIGASHIMARU SHOYU Co. (Tatsuno, Japan).

For both the Miso soup and Sumashi soup, we prepared the four specimens for each experimental run at once, and kept these at approximately $90^{\circ} \mathrm{C}$ using a thermal container equipped with a thermostatic controller. Prior to serving to participants, the salt concentration of each soup was monitored using a salt-concentration meter (PAL-SALT; a product of ATAGO Co., Tokyo, Japan). We utilized known concentrations of $\mathrm{NaCl}$ solutions $(0.5 \%, 0.7 \%$, and $0.9 \%)$ as a reference. The samples were served in paper cups at $55^{\circ} \mathrm{C}-60^{\circ} \mathrm{C}$ to the panelists, and the tasting process progressed from low (0\%) to high (3\%) soup stock consistency. 


\subsection{Panels}

The panels were composed of untrained individuals. Specifically, they were female students, aged 20 to 22, who were attending Prefectural University of Hiroshima. A total of 45 panelists assessed the flavor of cookies with or without bean curd lees, and 95 panelists evaluated the taste of Miso soup and Sumashi soup. For the cookie experiment, 21 participants composed panel A, and 24 formed panel B. Most of the members of panel A $(n=15)$ were assigned to be part of the pilot study, in which we tested the questionnaire with the 13 contrasting pairs of adjectives. The other 6 participants from panel A and all of the members of panel B $(n=24)$ evaluated the flavor of cookies with or without bean curd lees. The panelists assigned to evaluate the taste of Miso soup and Sumashi soup were assigned to groups as follows: 16 in panel C, 15 in panel D, 17 in panel E, 16 in panel F, 15 in panel $\mathrm{G}$, and 16 in panel $\mathrm{H}$. The participants in panels C, D, and E evaluated the taste of Miso soup, while those in panels F, G, and H assessed the taste of Sumashi soup. No participants participated in multiple components of the study.

\subsection{Sensory Questionnaire for Assessing the Flavor of Cookies with and without Bean Curd Lees}

As the sensory questionnaire was a key element of our study, we assigned most of panel A to the pilot study, where we tested the impression items, i.e., the 13 contrasting pairs of adjectives. We first screened five judges from the member constituents of panel $\mathrm{A}$, and asked these judges to join a discussion where the goal was to screen 25 levels of contrasting pairs of adjectives after tasting a cookie either with or without bean curd lees. After this screening process was complete, we picked out an additional five judges from the remaining panelists in panel A. These individuals were asked to taste a cookie with bean curd lees and assign the following threshold values (scores) for each adjective pair, one at a time: 0- "unfavorable or unsuitable"; 1 - "preferable if forced to choose"; or 2- "favorable or suitable". Similarly, the final five participants in panel A were asked to taste a cookie without bean curd lees, and then assign the above-mentioned threshold values to each adjective pair, individually. In this way, the following 13 impression items comprising contrasting adjective pairs were determined: delicious - not delicious, plain - rich, proper sweetness - less sweet, harmonious - inharmonious or watery, good aftertaste - bad aftertaste, moist - dry, smooth - coarse, fluffy - tough, soft - hard, chewy - not chewy, melting - not melting, flavorful - not flavorful, a little tea flavoring - non tea flavoring.

In our questionnaire assessment, the 13 descriptors in the questionnaire were scored on an 11-point scale ( -5 to +5 ), with 0 as the middle score. There were no symbolic representations associated with the numbers, as in the Likert scale (Likert 1932). In each experimental run, the questionnaire assessment (sensory test) was conducted twice: 1) once for a cookie with bean curd lees; and 2) once for a cookie without bean curd lees.

In each experimental run, the scores recorded in the first inquiry were subtracted from the relevant values in the second (the first minus the second) for each panelist. For each descriptor, we calculated the total sum of the difference in the scores between the first- and second-inquiry in the panel. We then evaluated the statistical significance of the 13 descriptors using Student's $t$-tests. Then, the mean difference in the score for each descriptor was plotted against the 13 descriptors, producing a sensory evaluation spectrum. The statistical significance of each descriptor was marked and scored as follows: a score was given an * (asterisk) and a significance score of 1 if the difference was significant such that $p<0.05$; a \pm and a significance score of 0.5 if the difference was significant such that $p=0.05-0.1$; and no symbol and a significance score of 0 if the difference was not significant such that $p \geq 0.1$. The sum of these scores was the total significance score $=\sum_{i=1}^{13}$ (significance score of the descriptor) $)_{i}$.

We used this value as an index of whether the relevant sensory spectrum could be considered to be statistically significant across the spectrograph (Sugawara, 2008; Sugawara et al., 2009a, 2013; Yamagata \& Sugawara, 2014). To confirm this, we used a sign test with $n=13$, corresponding to the number of descriptors used in our sensory test. We found that the spectra could be expected to reach significance $(p<0.05)$ when the number of descriptors regarded as significant at a probability value of $p<0.05$ (according to the $t$-test) was greater than 10 out of the 13 descriptors. In contrast, when this value was less than 3 , the null hypothesis could be rejected.

\subsection{Sensory Questionnaire for Assessing the Taste of Miso Soup and Sumashi Soup as a Function of Salt Concentration and Sop Stock Consistency}

As in the previous section, the taste of Miso soup was evaluated using the following 13 contrasting pairs of ad- 
jectives: mild taste - harsh taste, proper taste - tasteless or strong taste, rich taste - poor taste, taste delicious taste unpalatable, heartfelt taste - shallow taste, good aftertaste - bad aftertaste, fair scent of Miso - nasty scent of Miso, relish flavor of Miso - unpleasant flavor of Miso, fair scent of soup stock - nasty scent of soup stock, relish flavor of soup stock - unpleasant flavor of soup stock, elegant - unrefined, wholehearted - empty, feel relief feel restless.

Similarly, the taste of Sumashi soup was assessed using the following 13 descriptors: mild taste - harsh taste, proper taste - tasteless or strong taste, rich taste - poor taste, taste delicious - taste unpalatable, heartfelt taste shallow taste, good aftertaste - bad aftertaste, fair scent of soy sauce - nasty scent of soy sauce, relish flavor of soy sauce - unpleasant flavor of soy sauce, fair scent of soup stock - nasty scent of soup stock, relish flavor of soup stock - unpleasant flavor of soup stock, elegant - unrefined, wholehearted - empty, feel relief - feel restless.

In the questionnaire assessment of both Miso soup and Sumashi soup, the 13 descriptors were scored on an 11-point scale $(-5$ to +5$)$ with 0 as the middle score, as with the assessment of cookie flavor. The questionnaire was administered four times for each panelist such that they tasted four soup varieties with a constant salty concentration (i.e., $0.5 \%, 0.7 \%$, or $0.9 \%$ ): once each for the specimens with $0 \%, 0.1 \%, 0.2 \%$, and $0.3 \%$ soup stock consistency.

We saw the need for a soup stock reference in our experimental design. Thus, we employed either the $0 \%$ (without soup stock) or $0.2 \%$ (ordinary amount) soup stock sample as a control. We then compared the differences in each descriptor for each panelist using the scores of the reference (the former minus the latter). Statistical evaluation of the descriptors was conducted using Student's $t$-tests. The statistical procedures were identical to those used for the assessment of cookie flavor.

\subsection{Ethical Statement}

This study complied with the Declaration of Helsinki for Medical Research involving Human Subjects and all participants provided informed written or verbal consent. This study was approved by the Ethics Committee of the Institution of Health Science, Prefectural University of Hiroshima, Japan.

\section{Results}

\subsection{Visualizing the Assessment of Cookie Flavor via Sensory Spectrograph}

Panels A $(n=21)$ and B $(n=24)$ participated in this part of the study.

The pilot study led to the compilation of the following 13 impression items: delicious - not delicious, plain rich, proper sweetness - less sweet, harmonious - inharmonious or watery, good aftertaste - bad aftertaste, moist - dry, smooth - coarse, fluffy - tough, soft - hard, chewy - not chewy, melting - not melting, flavorful - not flavorful, a little tea flavor - non tea flavor.

The other members of panel A $(n=6)$ and all of panel B $(n=24)$ assessed the flavor of cookies with or without bean curd lees, which we then expressed using a sensory spectrograph. To measure the flavor of cookies with or without bean curd lees, the 13 descriptors in the questionnaire were scored on an 11-point scale ( -5 to $+5)$, with 0 as the middle score. Inquiry assessment, either practiced by panel A $(n=6)$ or panel B $(n=24)$, was conducted twice. The first inquiry was for cookies with bean curd lees, while the second one was for cookies without. The scores recorded in the second inquiry were subtracted from the scores obtained in the first inquiry (the first minus the second). Figure 1 shows a summary of the responses of the participants $(n=30)$.

Figure 1(a) shows the mean-deviation profile for the responses of the participants $(n=30)$. Here, shaded bars represent the mean \pm deviation profile for the first inquiry of cookie flavor for cookies with bean curd lees. The open bars represent the mean-deviation profile of the second inquiry of cookie flavor, which centered on cookies without bean curd lees. Visual observation of the figure indicates that the participants gave the two different types of cookies different ratings, i.e., they had a different taste. However, the standard deviation values in both inquiries were remarkably large relative to the means. Thus, these differences were statistically non-significant. Several factors may have led to this result: a lack of necessary mastery of sensory tests; absence of interest in the sensory target; a shortage of sensitivity to the stimuli; susceptibility to fatigue; and so on.

In contrast, the obtained sensory spectrograph (Figure 1(b)) reveals nuanced details. In this figure, the mean of the impression difference between the first and second inquiry (the first minus the second) was plotted against the setting semantic impression descriptors. Here, the statistical significance of each impression descriptor was 
(a)

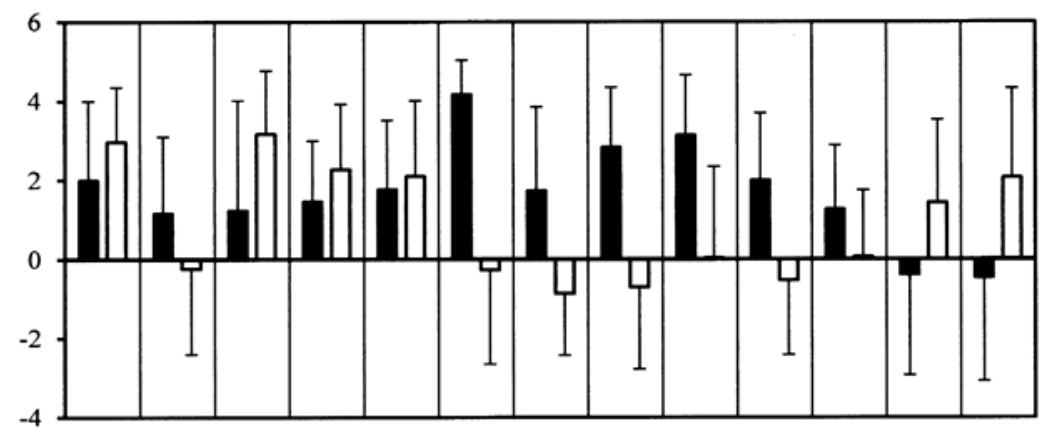

(b)

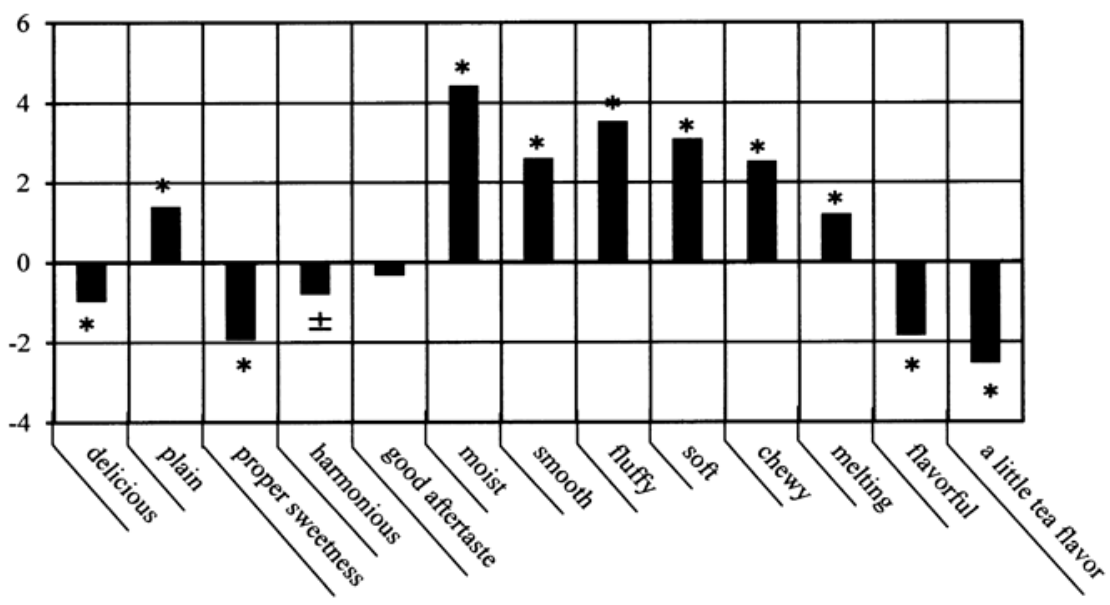

\begin{abstract}
Figure 1. Visualizing a flavor assessment of cookies with or without bean curd lees via sensory spectrograph. (a) The mean \pm standard deviation profile resulting from the sensory questionnaire assessment $(n=30)$; and (b) the profile in terms of a sensory spectrograph. The sensory test was conducted twice: once to assess the taste of cookies with bean curd lees; and once to test the taste of cookies without bean curd lees. Shaded bars represent the first inquiry, while open bars stand for the second inquiry. The difference in the mean scores between the first and the second inquiry was plotted against the 13 impression descriptors as a bar graph (sensory spectrograph). The statistical significance evaluated by $t$-test of each descriptor was marked as follows: * indicates that the itemwas regarded significant as per $p<0.05$; \pm , if regarded significant with $p=0.05-0.1$; and unmarked, if $p \geq 0.1$.
\end{abstract}

marked and scored in each sensory spectrograph as follows: 1.0 for items denoted with *; 0.5 for items denoted with \pm ; and 0.0 for items that are unmarked. The summation of these scores produces the total significance score ( $\sum_{i=1}^{13}$ (significance score of descriptor $)_{\mathrm{i}}$ ). In the spectrograph in Figure 1(b), this value was calculated to be 11.5.

This value can be used as an index of the statistical significance of a specific spectrum across the obtained spectrographs (Sugawara, 2008; Sugawara et al., 2009a, 2013; Yamagata \& Sugawara, 2014). As mentioned above, a sign test with $n=13$, which corresponds with the number of descriptors used in our sensory test, would enable us to evaluate the changes in each spectrum: 1$)$ it would be expected to reach significance $(p<0.05)$ when the value of the total significance score that corresponds to the number of descriptors regarded as significant at a probability value of $p<0.05$ (according to the $t$-test) was greater than 10 out of the 13 descriptors; 2) if this value was less than 3, the null hypothesis could be rejected. Thus, the trend shown in Figure 1(b) could be regarded as statistically significant $(p<0.05)$.

In connection with this issue, it is relevant to question how many panelists per group would be required to identify and quantify specific sensory attributes, if using untrained (inexperienced) individuals in this sensory study.

Figure 2 shows the results of our attempts to look for changes in the shape and total significance scores of the 
spectrographs, when artificially varying the number of panelists from $n=5$ to $n=30$ using computer software. For this purpose, we first combined all of our sensory test data from both panel A $(n=6)$ and panel B $(n=24)$. We then carried out a sequence of computer-generated graphic experiments, in which the number of participants could be varied from $n=5$ to $n=30$. The selection of participants was randomized using a table of randomized numbers.

Figure 2(a) shows the resulting sensory spectrograph for a randomly selected participant group with $n=5$. Figure 2(b) shows a spectrograph generated when the data from an additional five participants were randomly selected from the remaining shuffled test data $(n=25)$, producing a group with $n=10$. Similarly, Figure 2(c) shows the spectrum for a group with $n=15$, Figure 2(d) shows the spectrum for $n=20$, Figure 2(e) shows the spectrum for $n=25$, and Figure 2(f) shows the spectrum for $n=30$. In the figure, the total significance scores were calculated as follows: 6.5 for the spectrum of $n=5$ (Figure 2(a)), 7.5 for the spectrum of $n=10$ (Figure 2(b)), 10.5 for the spectrum of $n=15$ (Figure 2(c)), 11.0 for the spectrum of $n=20$ (Figure 2(d)), 11.0 for the spectrum of $n=25$ (Figure 2(e)), and 11.5 for the spectrum of $n=30$ (Figure 2(f)). The total significance scores of the spectra were greater than 10, excluding the cases of Figure 2(a) $(n=5)$ and Figure 2(b) $(n=10)$. Thus, if the number of participants were greater than 15, we would obtain satisfactory-to-good agreement in terms of reproducibility, consistency with sensory attributes in terms of shape, and statistical significance for the

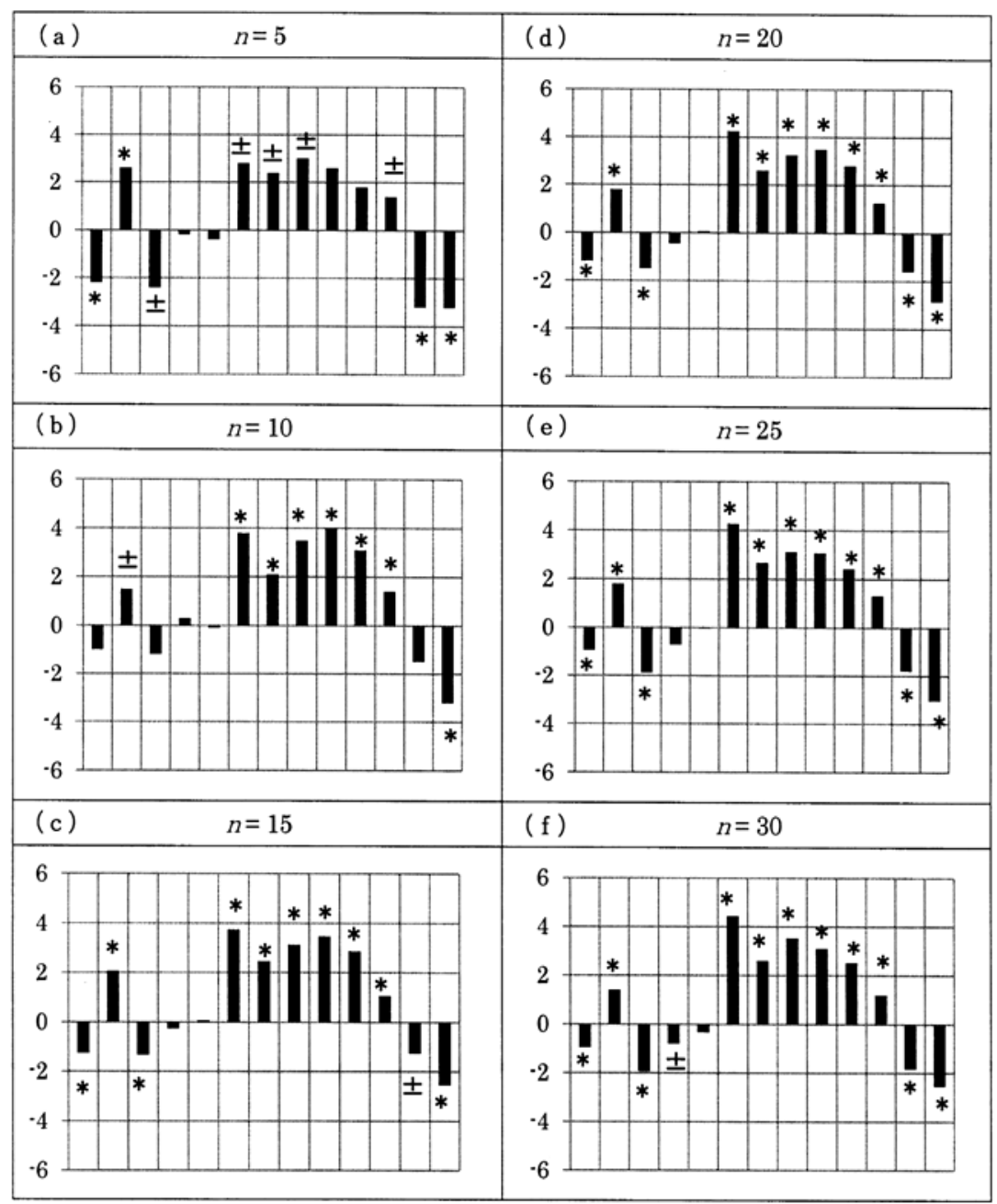

Figure 2. Changes in the shape and total significance score when the number of panelists was varied from $n=5$ to $n=30$. In a given spectrograph, the total significance score is defined as the total sum of the statistical scores of each descriptor as follows: 1.0 for items denoted with *; 0.5 for items denoted with \pm ; and 0.0 for items that are unmarked. 
obtained sensory spectrographs (Figure 3).

We speculate that the spectrograph in Figure 1(b) (or Figure 2(f)) might belong in the third "miscellaneous" type of our classification system, in which half of the significant descriptors were positive and the other half negative.

\subsection{Visualizing the Assessment of Miso Soup and Sumashi Soup Flavor via a Sensory Spectrum}

Under equivalent conditions to those established in above section, we attempted to visualize the assessment of Miso soup and Sumashi soup taste (Table 1) as a function of different salt concentrations and soup stock consistencies. As described, the taste of Miso soup or Sumashi soup was evaluated using 13 descriptors that were identified through a screening process. For Miso soup, these were mild taste - harsh taste, proper taste - tasteless or strong taste, rich taste - poor taste, taste delicious - taste unpalatable, heartfelt taste - shallow taste, good aftertaste - bad aftertaste, fair scent of Miso - nasty scent of Miso, relish flavor of Miso - unpleasant flavor of Miso, fair scent of soup stock - nasty scent of soup stock, relish flavor of soup stock - unpleasant flavor of soup stock, elegant - unrefined, wholehearted - empty, and feel relief - feel restless. For Sumashi soup, these were mild taste - harsh taste, proper taste - tasteless or strong taste, rich taste - poor taste, taste delicious - taste unpalatable, heartfelt taste - shallow taste, good aftertaste - bad aftertaste, fair scent of soy sauce - nasty scent of soy sauce, relish flavor of soy sauce - unpleasant flavor of soy sauce, fair scent of soup stock - nasty scent of soup stock, relish flavor of soup stock - unpleasant flavor of soup stock, elegant - unrefined, wholehearted - empty, and feel relief - feel restless.

Three different panels of participants, panel C $(n=16)$, D $(n=15)$, and E $(n=17)$ evaluated the Miso soup, while Panels F $(n=16), \mathrm{G}(n=15)$, and H $(n=16)$ assessed the Sumashi soup. For both the Miso soup and Sumashi soup, a sensory questionnaire with the 13 descriptors was completed four times in each experimental run for each panel: once each for the specimens with $0 \%, 0.1 \%, 0.2 \%$, and $0.3 \%$ soup stock consistency.

In our experimental design, we decided to identify an eligible reference for constructing a sensory spectrograph. The candidates for a reference were the specimens with $0 \%$ (without soup stock) or $0.2 \%$ (ordinary amount) soup stock consistency.

With these two samples as references, Figures 4-7 summarize the obtained sensory spectrographs for the taste assessment of the 12 varieties of Miso and Sumashi soup. For both Miso soup and Sumashi soup, Figure 4 and Figure 6 are representative, and show the result of fixing the sensory test data around $0 \%$ (without soup stock) soup stock consistency as a reference. This is also the case for Figure 5 and Figure 7, except that the sensory test data was fixed to reflect a reference of $0.2 \%$ (ordinary quantity used) soup stock consistency.

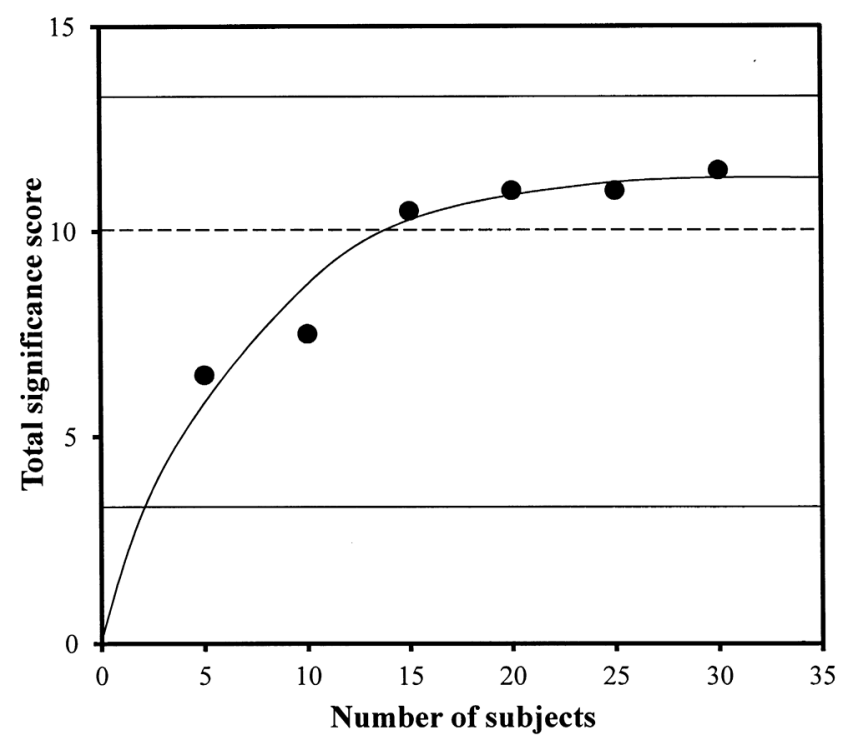

Figure 3. Plot of the total significance scores as a function of the number of panelists $(n=5-30)$. The details are identical to those in Figure 2. 
Figure 4 shows a summary of the assessment of the taste of the 12 varieties of Miso soup in a sensory spectrograph, while employing the sensory test data for $0 \%$ (without soup stock) soup stock consistency as a reference. As a function of different salt concentrations and soup stock consistencies, each spectrograph demonstrates an upward (positive) shape. The descriptors regarded as significant by a $t$-test had a positive value and are shown above the horizontal axis.

This signifies a positive (or favorable) inclination toward the taste of a given sample against the reference sensory test data ( $0 \%$ soup stock consistency) in regard to the "positive" descriptors (i.e., "mild taste", "proper taste", "rich taste", "taste delicious", "heartfelt taste", etc.). With respect to the total significance scores for these spectrographs, almost all samples were significant for more than 10 out of 13 descriptors, with the exception of Figure 4(e), in which this value was 9.0, just below 10 .

Figure 5, in turn, gives a summary of the taste appraisal for the 12 varieties of Miso soup when we adopted the sensory test data to a reference of $0.2 \%$ (ordinary amount used) soup stock consistency. The obtained findings demonstrate a downward (negative) spectrograph against the reference, and the descriptors regarded as significant by a $t$-test had a negative value and were shown below the horizontal axis. This signifies a negative (or unfavorable) "taste" of a given sample against the reference sensory test data in regard to the negative descriptors (i.e., "harsh taste”, "tasteless or strong taste”, "poor taste”, "taste unpalatable”, "shallow taste”, etc.). With respect to the total significance scores for the obtained spectrographs, almost all cases were insignificant for less than 4.5 out of 13 descriptors, with the exception of the spectrographs in Figure 5(a), Figure 5(d), and Figure 5 (g). In regard to these exceptional cases, the values of the total significance score were 11.5 for Figure 5(a), 9.5 for Figure 5(d), and 12.0 for Figure 5(g). Accordingly, a typical downward (negative) inclination was generally statistically significant in these spectrographs. However, the value in Figure 5(d) was an exception, at just below 10.

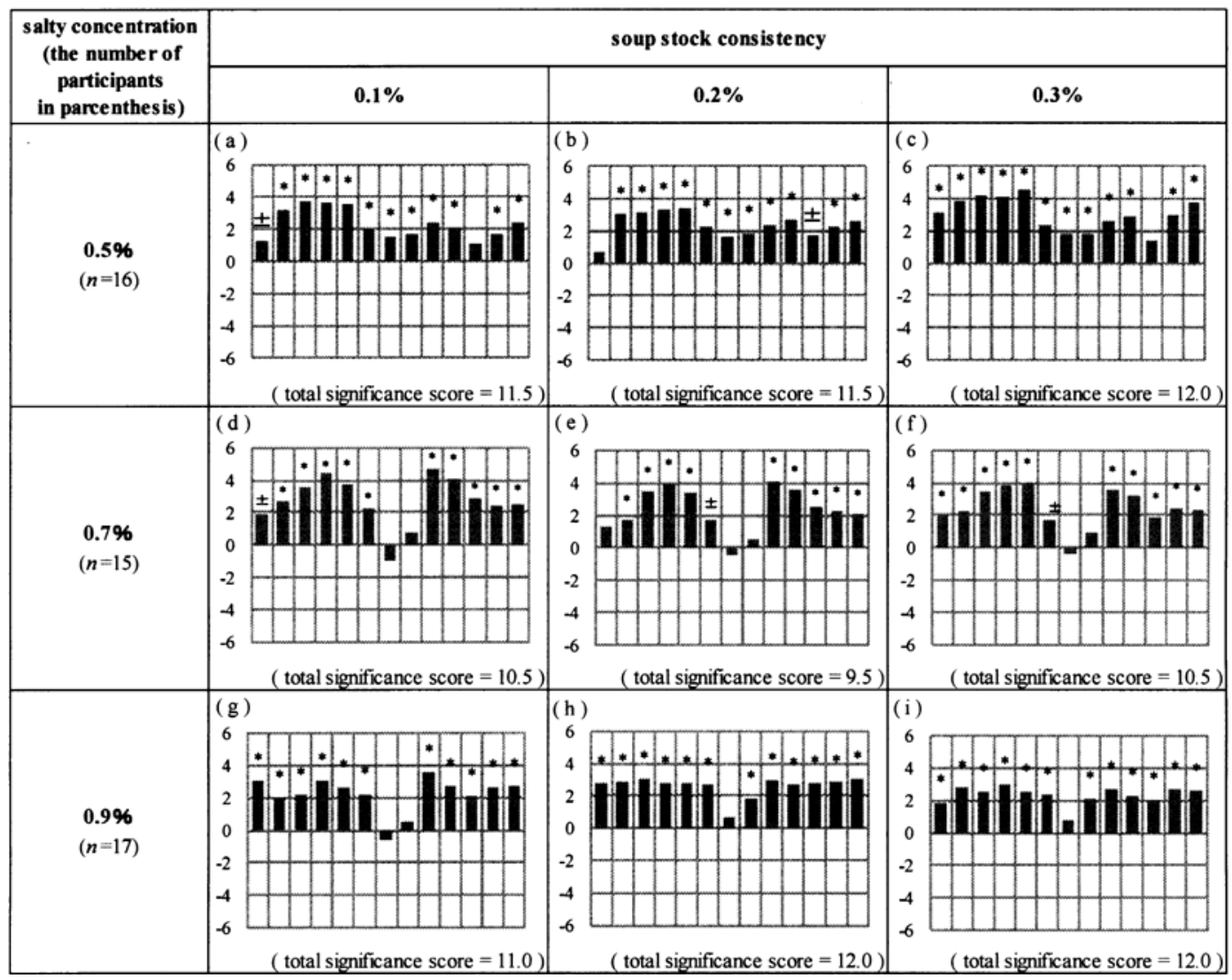

Figure 4. Sensory spectrograph showing summary of the verbal responses of the participants regarding 12 varieties of Miso soup (Table 1(a)) as a function of different salt concentrations and soup stock consistencies. In constructing the sensory spectrographs in the figure, we employed the sensory test data for $0 \%$ soup stock consistency (without soup stock) as a reference. 


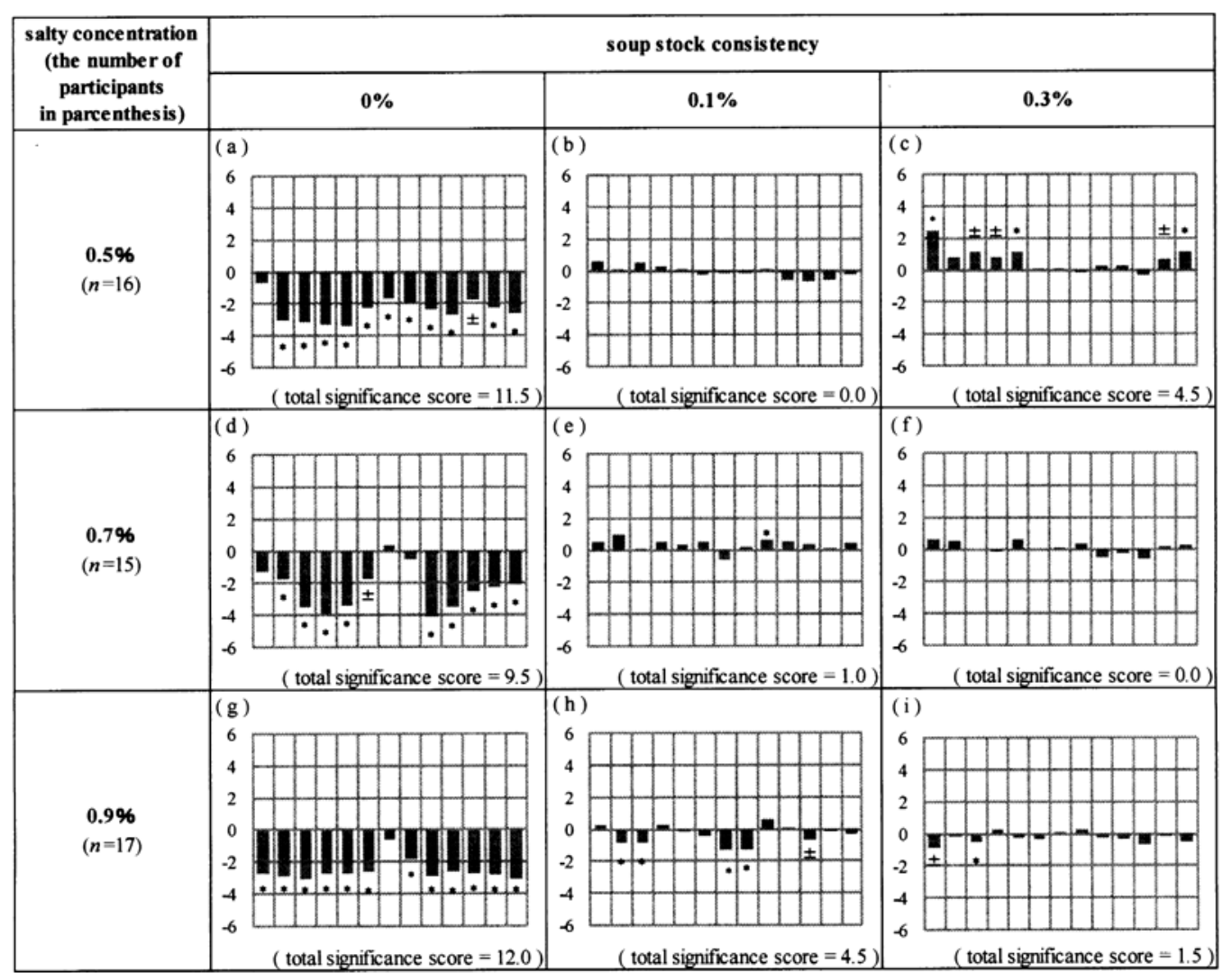

Figure 5. Sensory spectrograph showing summary of the verbal responses of the participants regarding 12 varieties of Miso soup (Table 1(a)) as a function of different salt concentrations and soup stock consistencies. In constructing the sensory spectrographs in the figure, we employed the sensory test data for $0.2 \%$ soup stock consistency (regular amount) as a reference.

Figure 6 and Figure 7 show a summary of the sensory spectrographs of taste assessment for the 12 varieties of Sumashi soup. The spectrographs in Figure 6 were constructed with the sensory test data for $0 \%$ (without soup stock) soup stock consistency as a reference, and the spectrographs in Figure 7 were derived using a sensory test data reference of $0.2 \%$ (ordinary quantity used). The obtained spectra for the 12 varieties of Sumashi soup were nearly the same as the results for the 12 varieties of Miso soup. As a function of different salt concentrations and soup stock consistencies, the spectra shown in Figure 6 represent an upward (positive) shape, while those in Figure 7 manifest a downward (negative) shape. In regard to the obtained total significance scores, all cases shown in Figure 6 were significant for more than 10 out of 13 descriptors. Expectedly, none of the spectra in Figure 7 reached statistical significance, and the obtained values were less than 4.5, although there were several exceptions: Figures 7(a)-(c) featured scores of 12.5 for (a), 10.5 for (b) and 10.5 for (c). This pattern was identical to that found for Miso soup (Figure 5).

\section{Discussion}

In the present study, we aimed to visualize the assessment of food flavor in terms of a sensory spectrograph. Accordingly, we conducted two studies. In the first study, we assessed the flavor of cookies with or without bean curd lees. In the second study, we evaluated the taste of Miso soup and Sumashi soup as a function of salt concentration and soup stock consistency.

We hoped that the obtained sensory spectra would exhibit satisfactory-to-good agreement with the data that could be obtained from the traditional descriptive sensory analysis in terms of reproducibility and consistency as well as statistical significance. This is because a traditional descriptive sensory analysis involves a group of trained individuals (generally 6 - 12) who identify and quantify specific sensory attributes with the goal of reproducibility and consistency (Drake, 2004, 2007; Lawless \& Heymann, 1988; Meilgaard et al., 1999). On the 


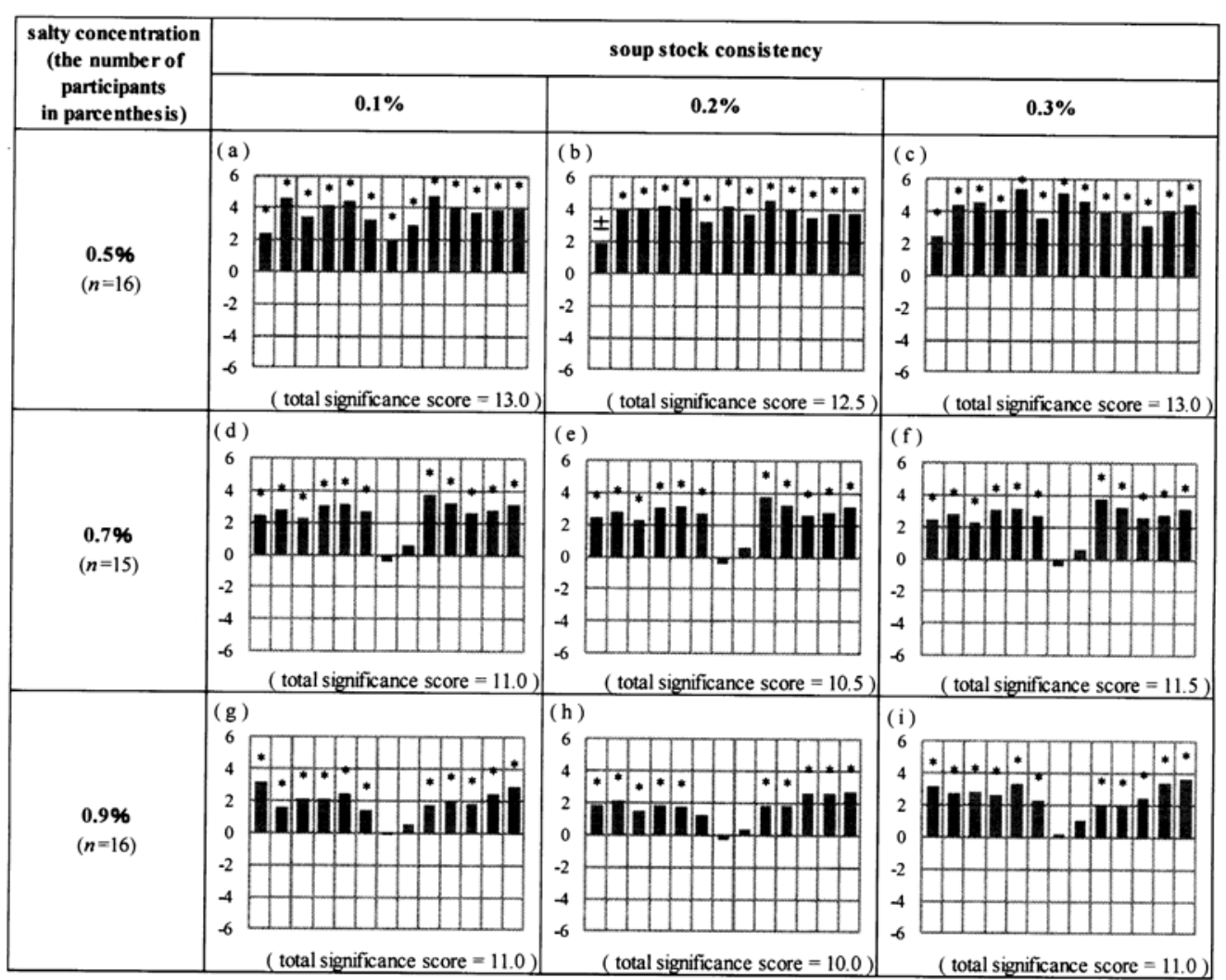

Figure 6. Sensory spectrograph showing summary of the verbal responses of the participants regarding 12 varieties of Sumashi soup (Table 1(b)) as a function of different salt concentrations and soup stock consistencies. In constructing the sensory spectrographs in the figure, we employed the sensory test data for $0 \%$ soup stock consistency (without soup stock) as a reference.

other hand, we chose to employ untrained individuals as panelists. Thus, our sensory study is subject to variations in the obtained data based on varying levels of participant interest in the sensory target, sensitivity to the stimuli, susceptibility to fatigue, and so on.

This was evident from the outcome of our first experiment, as shown in Figure 1(a), wherein the standard deviation of the responses both in the first and second questionnaires for assessing the flavor (taste) of cookies with and without, respectively, bean curd lees was remarkably large compared with the values of the mean for the 13 descriptors studied. When considering the large standard deviation values against the values of the mean, these means were statistically non-significant.

Regardless, the outcome of the first experiment (Figure 1(b)) seemed to demonstrate that we had represented sensory attributes at a level of quality that was equal to that of the traditional (conventional) descriptive sensory analyses. We plotted the mean of the difference in the scores between the first inquiry (for assessing the flavor of cookies with bean curd lees) and the second inquiry (for evaluating the flavor of cookies without bean curd lees) per participant in each panel (the first minus the second) against the 13 setting semantic impression descriptors as a bar graph (sensory spectrograph). The statistical significance of each descriptor was marked and scored as follows: 1.0 for items denoted with *; 0.5 for items denoted with \pm ; and 0.0 for items that are unmarked. This value was calculated as 11.5 (Figure 1(b)), indicating that the spectrograph reached statistical significance. As mentioned previously, this value should be $>10$ for the 13 descriptors if the obtained sensory spectrum is significant $(p<0.05)$.

In this context, we conducted a series of computer-generated graphics experiments while taking the sensory test data attained from both panels A $(n=6)$ and B $(n=24)$ regarding cookie taste assessment as the foundation of this work (Figure 2 and Figure 3). Figure 2 shows a summary of the changes in the shape and characteristics of the total significance score of the individual spectrographs obtained by varying the number of panelists from $n$ $=5$ to $n=30$. The obtained data (Figure 2) demonstrate satisfactory-to good agreement concerning the obtained 


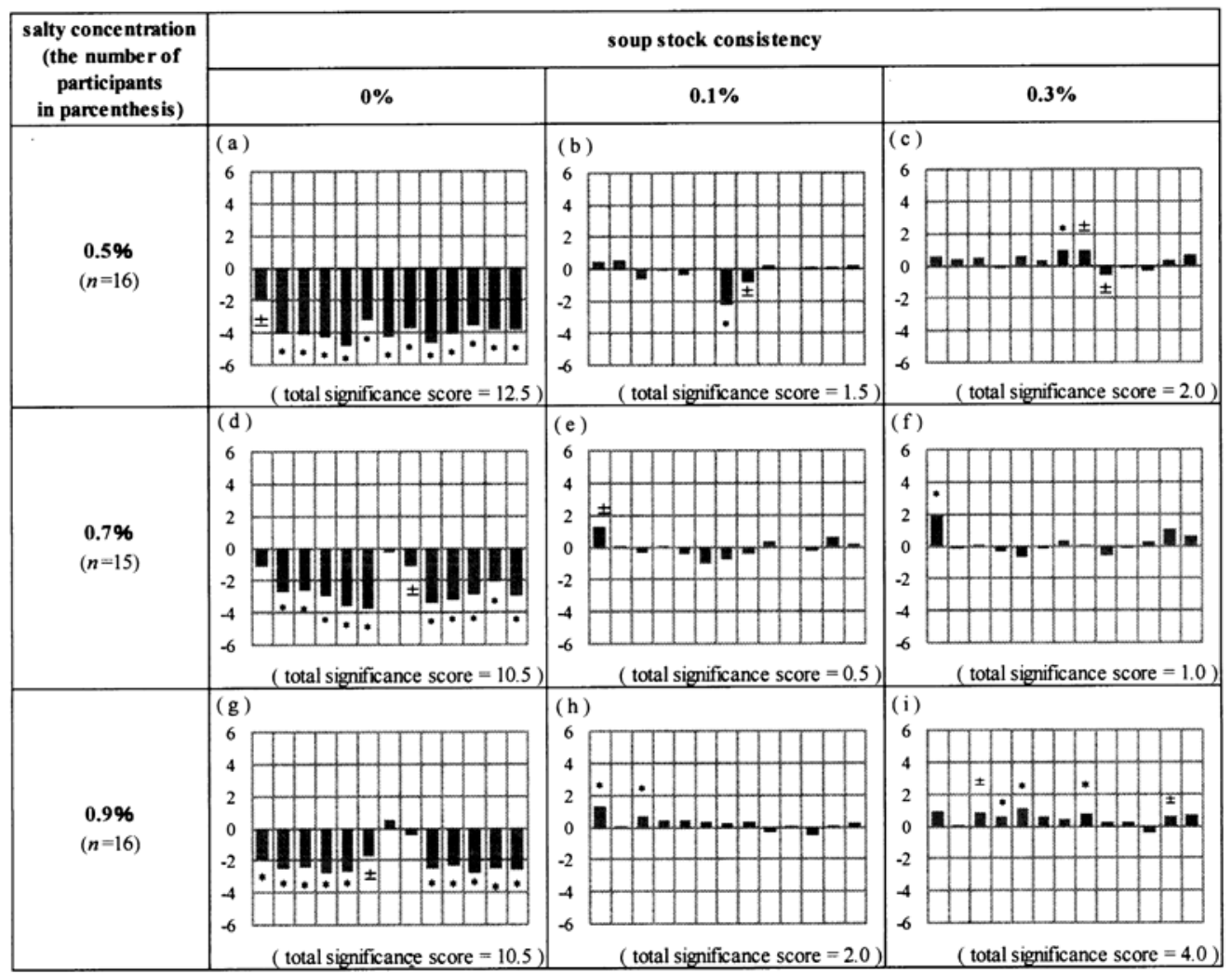

Figure 7. Sensory spectrograph showing summary of the verbal responses of the participants regarding 12 varieties of Sumashi soup (Table 1(b)) as a function of different salt concentrations and soup stock consistencies. In constructing the sensory spectrographs in the figure, we employed the sensory test data for $0.2 \%$ soup stock consistency (regular amount) as a reference.

sensory spectra with respect to reproducibility and consistency, both in terms of the shape and characteristics of the total significance score. Figure 3 focuses on the changes in the total significance scores as a function of the number of panelists $(n=5-30)$. We found that the total significance scores were all above 10 in the spectrographs for which the number of panelists was greater than $15(n=15)$. This means that we could increase the number of panelists involved in our study to dramatically improve the chances of significance. Note that the value of a total significance score can be expected to be greater than 10 if the spectrum is regarded as significant $(p<0.05)$.

In our second study, we used groups of panelists that were larger than 15. Figures 4-7 show the results of a taste assessment for Miso soup and Sumashi soup as a function of salt concentration and soup stock consistency.

Figure 4 shows a representative set of spectrographs for a taste assessment of Miso soup. In the figure, each spectrograph manifests an upward (positive) spectrum, such that the descriptors regarded as significant by a $t$-test had a positive value and were shown above the horizontal axis. This signifies a positive (or favorable) impression regarding the taste of a given sample when compared with the taste of $0 \%$ (without soup stock) soup stock consistency sample, with respect to the positive descriptors (i.e., "mild (taste)", "proper (taste)", "rich (taste)", "(taste) delicious", "heartfelt (taste)", etc.). Moreover, the spectra reached statistical significance ( $p<$ 0.05). Almost all total significance score vales were greater than 10 for the 13 descriptors, although Figure 4(e) and Figure 4(h), in which the values were 9.0 for (e) and 9.5 for (h), were exceptions. An identical pattern was seen in the relevant spectra concerning a given sample of Sumashi soup (Figure 6).

For the sensory spectra shown in Figure 5 and Figure 7, for both Miso soup and Sumashi soup, our sensory spectrographs indicate some practical potentialities. First, the six spectra in the first (right-hand) column of each row in the figures show a downward (negative) spectrograph, in which negative values appear below the axis. This suggests an unfavorable (negative) impression regarding the taste of a given sample when compared with the taste of $0.2 \%$ (ordinary quantity used) soup stock consistency as a reference in regard to the negative de- 
scriptors (i.e., "harsh (taste)”, “tasteless or strong (taste)”, “poor (taste)”, “(taste) unpalatable”, “shallow (taste)”, etc.). Additionally, five of the six spectra were regarded as significant $(p<0.05)$, because the values of the total significance scores were calculated be greater than 10. In the middle of these figures, we can see spectra of a "miscellaneous" type, as half of the significant descriptors are positive and the other half negative, although the values of the total significance scores are less than 4.5. Moreover, when looking at the six right-hand spectra, each shows a positive inclination regarding the impression of the taste of a given specimen, as the values of the total significance scores were less than 4.5 .

We believe that the above-mentioned phenomenon relates to the sensory characteristics shown in Figure 1(b) (or Figure 2(f)). It seems that the shape of the sensory spectrograph in Figure 1(b) (or Figure 2(f)) can be classified as "miscellaneous" type. However, the nutritional value of a cookie fortified with bean curd lees is greater, as it contains 2.3 times the dietary fiber, $10 \%$ fewer calories, 1.5 times the calcium content, and 1.5 times the amount of iron compared with a cookie without bean curd lees. Thus, there are factors that may not be considered by participants when evaluating the cookies, although these could be included in future evaluations.

We are currently developing an application to use our sensory spectrum method to characterize the taste of commercially available foods, especially commodities that have been developed in recent years for elderly persons. Our findings imply that sensory spectrographs can be used to access the finer nuances of taste profiles. Thus, this method of sensory profiling has practical applications for assessing food flavors. Indeed, this method may represent a new and different approach to the assessment of flavor from the view of semantics.

\section{Conclusions}

Based on the experiences conducting sensory profiling of aromas, in this paper, we sought to assess the perception of food flavor via sensory spectrograph: a bar graph where the mean of the impression is plotted against descriptors of the setting for the semantic impression.

For this objective, we conducted two studies: the first study assessed the flavor of cookies with or without bean curd lees; and the second study evaluated the taste of Miso soup and Sumashi soup as a function of salt concentration and soup stock consistency.

As a result, the obtained data of both studies demonstrate that our sensory spectrum method could represent sensory attributes at a level of quality that was equal to that of the traditional (conventional) descriptive analyses in terms of reproducibility and consistency, although we chose to employ untrained individual as panellists.

Specifically, a series of computer-generated graphics experiments in the first study while taking the sensory test data attained from both panels A $(n=6)$ and B $(n=24)$ regarding cookie taste assessment as the foundation of this work revealed that we would obtain satisfactory-to-good agreement in the shape and characteristics of the statistical significance, if the number of participants were greater than 15.

The outcomes in the second study, in which we used groups of panellists that were larger than 15, suggest that sensory spectrographs could access the finer nuances of taste profiles, if we can choose an eligible reference for constructing a sensory spectrograph.

Thus, we believe that our sensory spectrum method may represent a new and different approach to the assessment of flavour from the view of semantics.

\section{References}

Coombs, C. H. (1964). Theory of Data. New York: John Wiley.

Drake, M. A. (2004). Defining Dairy Flavors. Journal of Dairy Science, 87, 777-784. http://dx.doi.org/10.3168/jds.S0022-0302(04)73221-X

Drake, M. A. (2007). Sensory Analysis of Dairy Foods. Journal of Dairy Science, 90, 4925-4937. http://dx.doi.org/10.3168/jds.2007-0332

Guilford, J. P. (1954). Psychometric Methods. New York: McGraw-Hill.

Kling, J. W., \& Riggs, L. A. (1972). Experimental Psychology. New York: Holt, Reinhart and Winston.

Lawless, H. T., \& Heymann, H. (1988). Sensory Evaluation of Food: Practices and Principals. New York: Chapman and Hall.

Likert, R. (1932). A Technique for the Measurement of Attitudes. Archives of Psychology, 140, 44-53.

Meilgaard, M. C., Giville, G. V., \& Carr, B. T. (1999). Sensory Evaluation of Techniques (3rd ed.). Boca Raton, FL: CRC 
Press. http://dx.doi.org/10.1201/9781439832271

Ohloff, G., \& Klein, E. (1962). Die absolute konfiguration des linalools durch verknuepfung mit dem pinansystem. Tetrahedron, 18, 37-42. http://dx.doi.org/10.1016/0040-4020(62)80021-0

Satoh, T., \& Sugawara, Y. (2003). Effects on Humans Elicited by Inhaling the Fragrance of Essential Oils: Sensory Test, Multi-Channel Thermometric Study and Forehead Surface Potential Wave Measurement on Basil and Peppermint. Analytical Sciences, 19, 139-146. http://dx.doi.org/10.2116/analsci.19.139

Stevens, S. S. (1951). Handbook of Experimental Psychology. New York: John Wiley.

Sugawara, Y. (2001). Odor Distinctiveness between Enantiomers of Linalool. Current Topics in Analytical Chemistry, 2, 201-210.

Sugawara, Y. (2008). Sensory Profiling for Verbal Responses in Perceived Odor Quality in Humans. In L. N. Piccard (Ed.), Biological Psychology: New Research (Chap. 4, pp. 145-165). New York: Nova Science Publishers.

Sugawara, Y., \& Kawasaki, M. (2000). Skin Temperature Change Elicited by Inhalation of the Fragrance of Essential Oils in Relation to Mental Work: Multi-Channel Thermometric Study on Basil and Peppermint. Journal of Home Economics of Japan, 51, 675-681.

Sugawara, Y., Fukui, H., Shigeoka, C., Hasegawa, R., \& Okimoto, A. (2006). Multichannel Thermometric Study of Temperature Changes in Humans While Inhaling Essential Oils. Flavour and Fragrance Journal, 21, 416-422. http://dx.doi.org/10.1002/ffj.1683

Sugawara, Y., Hara, C., Aoki, T., Sugimoto, N., \& Masujima, T. (2000). Odor Distinctiveness between Enantiomers of Linalool: Difference in Perception and Responses Elicited by Sensory Test and Forehead Surface Potential Wave. Chemical Senses, 25, 77-84. http://dx.doi.org/10.1093/chemse/25.1.77

Sugawara, Y., Hara, C., Tamura, K., Fujii, T., Nakamura, K., Masujima, T., \& Aoki, T. (1998a). Sedative Effect on Humans of Inhalation of Essential Oil of Linalool: Sensory Evaluation and Physiological Measurements Using Optically Active Linalools. Analytica Chimica Acta, 365, 293-299. http://dx.doi.org/10.1016/S0003-2670(97)00639-9

Sugawara, Y., Hino, Y., Kawasaki, M., Hara, C., Tamura, K., Sugimoto, N., Yamanishi, U., Miyauchi, M., Masujima, T., \& Aoki, T. (1999). Alteration of Perceived Fragrance of Essential Oils in Relation to Type of Work: A Simple Screening Test for Efficacy of Aroma. Chemical Senses, 24, 415-421. http://dx.doi.org/10.1093/chemse/24.4.415

Sugawara, Y., Maruyama, S., Nakagawa, N., Yamada, E., Seto, M., Sumihiro, S., Aoi, N., Nishimoto, M., Kobayashi, Y., \& Hirano, M. (2008). Verbal and Non-Verbal Responses to Odorants in Humans While Inhaling the Fragrance of Peppermint and Spearmint Essential Oils and Linalool. International Journal of Essential Oil Therapeutics, 2, 111-121.

Sugawara, Y., Nishimoto, M., Kobayashi, Y., Hasegawa, R., Okimoto, A., \& Aoki, T. (2003). Repeatability of the Measure Required for Perceptional Changes of the Fragrance of Essential Oils Was Tested in Terms of Sensory Evaluation Spectrums. Bull. Fac. Human Life Environ. Sci. Hiroshima Women's Univ., 9, 21-36.

Sugawara, Y., Shigetho, A., Yoneda, M., Tuchiya, T., Matumura, T., \& Hirano, M. (2013). Relationship between Mood Change, Odour and Its Physiological Effects in Humans While Inhaling the Fragrances of Essential Oils as Well as Linalool and Its Enantiomers. Molecules, 18, 3312-3338. http://dx.doi.org/10.3390/molecules18033312

Sugawara, Y., Shigetho, A., Yoneda, M., Tuchiya, T., Matumura, T., \& Hirano, M. (2015a). Relationship between Mood Change, Odour and Its Physiological Responses in Humans in Terms of the Sensory Evaluation Spectrum. Psychology, 6, 965-988. http://dx.doi.org/10.4236/psych.2015.68095

Sugawara, Y., Shigetho, A., Yoneda, M., Tuchiya, T., Matumura, T., \& Hirano, M. (2015b). Versatile Psychophysiological Potencies of Essential Oils, when Seen as a Function of Behavioral Task Assigned to the Participants after Inhalation. International Journal of Social Science Studies, 3, 94-113. http://dx.doi.org/10.11114/ijsss.v3i6.1129

Sugawara, Y., Sugimoto, C., \& Minabe, S. (2009a). Sensory Evaluation of the Deodorizing Efficacy of a Titanium OxideType Deodorant. Journal of Home Economics of Japan, 60, 353-362.

Sugawara, Y., Sugimoto, C., Minabe, S., Iura, Y., Okazaki, M., Nakagawa, N., Seto, M., Maruyama, S., Hirano, M., \& Kitayama, I. (2009b). Use of Human Senses as Sensors. Sensors, 9, 3184-3204. http://dx.doi.org/10.3390/s90503184

Sugawara, Y., Tomota, T., \& Tamura, K. (1998b). Perceived Fragrance of Essential Oils in Relation to Type of Work. Journal of Home Economics of Japan, 49, 1281-1290.

Torgerson, W. S. (1958). Theory and Methods of Scaling. New York: John Wiley.

Yamagata, Y., \& Sugawara, Y. (2014). Sensory Evaluation Spectrum Method as a Descriptive Sensory Analysis. Psychology, 5, 1591-1610. http://dx.doi.org/10.4236/psych.2014.514170 